\title{
Inverse eigenvalue problems for a discontinuous Sturm-Liouville operator with two discontinuities
}

\section{Yalçın Güldü*}

\section{"Correspondence:}

yguldu@cumhuriyet.edu.tr Department of Mathematics, Faculty of Sciences, Cumhuriyet University, Sivas, 58140, Turkey

\section{Springer}

\begin{abstract}
In this paper, we consider a discontinuous Sturm-Liouville operator with parameter-dependent boundary conditions and two interior discontinuities. We obtain eigenvalues and eigenfunctions together with their asymptotic approximate formulas. Then, we give some uniqueness theorems by using Weyl function and spectral data, which are called eigenvalues and normalizing constants for solution of inverse problem.

MSC: Primary 34A55; secondary 34B24; 34L05

Keywords: Sturm-Liouville problem; eigenvalues; eigenfunctions; transmission conditions; Weyl function
\end{abstract}

\section{Introduction}

It is well known that the theory of Sturm-Liouville problems is one of the most actual and extensively developing fields of theoretical and applied mathematics, since it is an important tool in solving many problems in mathematical physics (see [1-4]). In recent years, there has been increasing interest in spectral analysis of discontinuous SturmLiouville problems with eigenvalue-linearly and nonlinearly dependent boundary conditions [1, 5-12]. Various physics applications of such problems can be found in $[1,3,4$, 13-19] and corresponding bibliography cited therein.

Some boundary value problems with discontinuity conditions arise in heat and mass transfer problems, mechanics, electronics, geophysics and other natural sciences (see [3] also [20-29]). For instance, discontinuous inverse problems appear in electronics for building parameters of heterogeneous electronic lines with attractive technical characteristics [20,30,31]. Such discontinuity problems also appear in geophysical forms for oscillations of the earth [32, 33]. Furthermore, discontinuous inverse problems appear in mathematics for exploring spectral properties of some classes of differential and integral operators.

Inverse problems of spectral analysis form recovering operators by their spectral data. The inverse problem for the classical Sturm-Liouville operator was studied first by Ambarsumian in 1929 [34] and then by Borg in 1945 [35]. After that, direct and inverse problems for Sturm-Liouville operator have been extended to so many different areas.

C 2013 Güldü; licensee Springer. This is an Open Access article distributed under the terms of the Creative Commons Attribution License (http://creativecommons.org/licenses/by/2.0), which permits unrestricted use, distribution, and reproduction in any medium, provided the original work is properly cited. 
We consider a discontinuous Sturm-Liouville problem $L$ with function $\rho(x)$

$$
l y:=-\rho(x) y^{\prime \prime}(x)+q(x) y(x)=\lambda y(x), \quad x \in\left[a, \delta_{1}\right) \cup\left(\delta_{1}, \delta_{2}\right) \cup\left(\delta_{2}, b\right]=\Omega,
$$

where

$$
\rho(x)= \begin{cases}\rho_{1}^{-2}, & a \leq x<\delta_{1}, \\ \rho_{2}^{-2}, & \delta_{1}<x<\delta_{2}, \\ \rho_{3}^{-2}, & \delta_{2}<x \leq b,\end{cases}
$$

and $\rho_{1}, \rho_{2}$, and $\rho_{3}$ are given positive real numbers; $q(x) \in L_{2}[\Omega, \mathbb{R}] ; \lambda \in \mathbb{C}$ is a complex spectral parameter; boundary conditions at the endpoints

$$
\begin{aligned}
& l_{1} y:=\lambda\left(\theta_{1}^{\prime} y(a)-\theta_{2}^{\prime} y^{\prime}(a)\right)-\left(\theta_{1} y(a)-\theta_{2} y^{\prime}(a)\right)=0, \\
& l_{2} y:=\lambda\left(\gamma_{1}^{\prime} y(b)-\gamma_{2}^{\prime} y^{\prime}(b)\right)+\left(\gamma_{1} y(b)-\gamma_{2} y^{\prime}(b)\right)=0
\end{aligned}
$$

with discontinuity conditions at two points $x=\delta_{1}, x=\delta_{2}$

$$
\begin{aligned}
& l_{3} y:=y\left(\delta_{1}+0\right)-\theta_{3} y\left(\delta_{1}-0\right)-\gamma_{3} y^{\prime}\left(\delta_{1}-0\right)=0, \\
& l_{4} y:=y^{\prime}\left(\delta_{1}+0\right)-\theta_{4} y\left(\delta_{1}-0\right)-\gamma_{4} y^{\prime}\left(\delta_{1}-0\right)=0, \\
& l_{5} y:=y\left(\delta_{2}+0\right)-\theta_{5} y\left(\delta_{2}-0\right)-\gamma_{5} y^{\prime}\left(\delta_{2}-0\right)=0, \\
& l_{6} y:=y^{\prime}\left(\delta_{2}+0\right)-\theta_{6} y\left(\delta_{2}-0\right)-\gamma_{6} y^{\prime}\left(\delta_{2}-0\right)=0,
\end{aligned}
$$

where $\theta_{i}, \gamma_{i}$ and $\theta_{j}^{\prime}, \gamma_{j}^{\prime}(i=1,6, j=1,2)$ are real numbers and

$$
\begin{aligned}
\alpha_{1} & =\left|\begin{array}{ll}
\theta_{3} & \gamma_{3} \\
\theta_{4} & \gamma_{4}
\end{array}\right|>0, \quad \alpha_{2}=\left|\begin{array}{ll}
\theta_{5} & \gamma_{5} \\
\theta_{6} & \gamma_{6}
\end{array}\right|>0, \quad \beta_{1}=\left|\begin{array}{ll}
\theta_{1}^{\prime} & \theta_{1} \\
\theta_{2}^{\prime} & \theta_{2}
\end{array}\right|>0 \quad \text { and } \\
\beta_{2} & =\left|\begin{array}{ll}
\gamma_{1}^{\prime} & \gamma_{1} \\
\gamma_{2}^{\prime} & \gamma_{2}
\end{array}\right|>0 .
\end{aligned}
$$

In the present paper, we construct a linear operator $T$ in a suitable Hilbert space such that problem (1)-(7) and the eigenvalue problem for operator $T$ coincide. We investigate eigenvalues and eigenfunctions together with their asymptotic behaviors of operator $T$. Besides, we study some uniqueness theorems according to Weyl function and spectral data, which are called eigenvalues and normalizing constants.

\section{Operator formulation and spectral properties}

We make known the inner product in the Hilbert space $H:=H_{1} \oplus \mathbb{C}^{2}$, where $H_{1}=$ $\left(L_{2}(\Omega),\langle\cdot, \cdot\rangle_{1}\right), \mathbb{C}$ denotes the Hilbert space of complex numbers and a self-adjoint operator $T$ defined on $H$ such that (1)-(7) can be dealt with as the eigenvalue problem of operator $T$. We define an inner product in $H$ by

$$
\begin{aligned}
\langle F, G\rangle:= & \alpha_{1} \alpha_{2} \rho_{1}^{2} \int_{a}^{\delta_{1}} f(x) \bar{g}(x) d x+\alpha_{2} \rho_{1}^{2} \int_{\delta_{1}}^{\delta_{2}} f(x) \bar{g}(x) d x+\rho_{3}^{2} \int_{\delta_{2}}^{b} f(x) \bar{g}(x) d x \\
& +\frac{\alpha_{1} \alpha_{2}}{\beta_{1}} f_{1} \bar{g}_{1}+\frac{1}{\beta_{2}} f_{2} \bar{g}_{2}
\end{aligned}
$$


for

$$
F=\left(\begin{array}{c}
f(x) \\
f_{1} \\
f_{2}
\end{array}\right) \in H, \quad G=\left(\begin{array}{c}
g(x) \\
g_{1} \\
g_{2}
\end{array}\right) \in H .
$$

Consider the operator $T$ defined by the domain

$$
\begin{aligned}
D(T)= & \left\{F \in H: f(x), f^{\prime}(x) \in A C_{\mathrm{loc}}(\Omega), l f \in H_{1}, l_{3}=l_{4}=l_{5}=l_{6}=0, f_{1}=\theta_{1}^{\prime} f(a)-\theta_{2}^{\prime} f^{\prime}(a)\right. \\
& \left.f_{2}=\gamma_{1}^{\prime} f(b)-\gamma_{2}^{\prime} f^{\prime}(b)\right\}
\end{aligned}
$$

such that TF := $\left(l f, \theta_{1} f(a)-\theta_{2} f^{\prime}(a),-\left(\gamma_{1} f(b)-\gamma_{2} f^{\prime}(b)\right)\right)$ for $F=\left(f, \theta_{1}^{\prime} f(a)-\theta_{2}^{\prime} f^{\prime}(a), \gamma_{1}^{\prime} f(b)-\right.$ $\left.\gamma_{2}^{\prime} f^{\prime}(b)\right) \in D(T)$ and also $l_{3}-l_{6}$ are satisfied for $f$.

Thus, we can rewrite the considered problem (1)-(7) in the operator form as $T F=\lambda F$.

Theorem 1 The operator $T$ is symmetric in $H$.

Proof Let $F, G \in D(T)$. By two partial integrations, we get

$$
\begin{aligned}
\langle T F, G\rangle= & \langle F, T G\rangle+\alpha_{1} \alpha_{2}\left(W\left(f, \bar{g}, \delta_{1}-0\right)-W(f, \bar{g}, a)\right) \\
& +\alpha_{2}\left(W\left(f, \bar{g}, \delta_{2}-0\right)-W\left(f, \bar{g}, \delta_{1}+0\right)\right)+W(f, \bar{g}, b)-W\left(f, \bar{g}, \delta_{2}+0\right) \\
& +\frac{\alpha_{1} \alpha_{2}}{\beta_{1}}\left(\theta_{1} f(a)-\theta_{2} f^{\prime}(a)\right)\left(\theta_{1}^{\prime} \bar{g}(a)-\theta_{2}^{\prime} \bar{g}^{\prime}(a)\right) \\
& -\frac{1}{\beta_{2}}\left(\gamma_{1} f(b)-\gamma_{2} f^{\prime}(b)\right)\left(\gamma_{1}^{\prime} \bar{g}(b)-\gamma_{2}^{\prime} \bar{g}^{\prime}(b)\right) \\
& -\frac{\alpha_{1} \alpha_{2}}{\beta_{1}}\left(\theta_{1} \bar{g}(a)-\theta_{2} \bar{g}^{\prime}(a)\right)\left(\theta_{1}^{\prime} f(a)-\theta_{2}^{\prime} f^{\prime}(a)\right) \\
& +\frac{1}{\beta_{2}}\left(\gamma_{1} \bar{g}(b)-\gamma_{2} \bar{g}^{\prime}(b)\right)\left(\gamma_{1}^{\prime} f(b)-\gamma_{2}^{\prime} f^{\prime}(b)\right),
\end{aligned}
$$

where by $W(f, g ; x)$, we denote the Wronskian of the functions $f$ and $g$ as

$$
f(x) g^{\prime}(x)-f^{\prime}(x) g(x)
$$

Since $f$ and $g$ satisfy the boundary conditions (2)-(3) and transmission conditions (4)(7), we obtain

$$
\begin{aligned}
\frac{\alpha_{1} \alpha_{2}}{\beta_{1}} & {\left[\left(\theta_{1} f(a)-\theta_{2} f^{\prime}(a)\right)\left(\theta_{1}^{\prime} \bar{g}(a)-\theta_{2}^{\prime} \bar{g}^{\prime}(a)\right)-\left(\theta_{1} \bar{g}(a)-\theta_{2} \bar{g}^{\prime}(a)\right)\left(\theta_{1}^{\prime} f(a)-\theta_{2}^{\prime} f^{\prime}(a)\right)\right] } \\
= & \alpha_{1} \alpha_{2} W(f, \bar{g}, a) \\
& \times \frac{1}{\beta_{2}}\left[\left(\gamma_{1} \bar{g}(b)-\gamma_{2} \bar{g}^{\prime}(b)\right)\left(\gamma_{1}^{\prime} f(b)-\gamma_{2}^{\prime} f^{\prime}(b)\right)-\left(\gamma_{1} f(b)-\gamma_{2} f^{\prime}(b)\right)\left(\gamma_{1}^{\prime} \bar{g}(b)-\gamma_{2}^{\prime} \bar{g}^{\prime}(b)\right)\right] \\
= & -W(f, \bar{g}, b) W\left(f, \bar{g}, \delta_{i}+0\right)=\alpha_{i} W\left(f, \bar{g}, \delta_{i}-0\right) .
\end{aligned}
$$

Thus, we have $\langle T F, G\rangle=\langle F, T G\rangle$, i.e., $T$ is symmetric. 
Lemma 1 Problem (1)-(7) can be considered as the eigenvalue problem of the symmetric operator $T$.

Corollary 1 All eigenvalues and eigenfunctions of problem (1)-(7) are real, and two eigenfunctions $\varphi\left(x, \lambda_{1}\right)$ and $\varphi\left(x, \lambda_{2}\right)$, corresponding to different eigenvalues $\lambda_{1}$ and $\lambda_{2}$, are orthogonal in the sense of

$$
\begin{aligned}
& \alpha_{1} \alpha_{2} \rho_{1}^{2} \int_{a}^{\delta_{1}} \varphi\left(x, \lambda_{1}\right) \varphi\left(x, \lambda_{2}\right) d x+\alpha_{2} \rho_{2}^{2} \int_{\delta_{1}}^{\delta_{2}} \varphi\left(x, \lambda_{1}\right) \varphi\left(x, \lambda_{2}\right) d x \\
& \quad+\rho_{3}^{2} \int_{\delta_{2}}^{b} \varphi\left(x, \lambda_{1}\right) \varphi\left(x, \lambda_{2}\right) d x \\
& \quad+\frac{\alpha_{1} \alpha_{2}}{\beta_{1}}\left(\theta_{1}^{\prime} \varphi\left(a, \lambda_{1}\right)-\theta_{2}^{\prime} \varphi^{\prime}\left(a, \lambda_{1}\right)\right)\left(\theta_{1}^{\prime} \varphi\left(a, \lambda_{2}\right)-\theta_{2}^{\prime} \varphi^{\prime}\left(a, \lambda_{2}\right)\right) \\
& +\frac{1}{\beta_{2}}\left(\gamma_{1}^{\prime} \varphi\left(b, \lambda_{1}\right)-\gamma_{2}^{\prime} \varphi^{\prime}\left(b, \lambda_{1}\right)\right)\left(\gamma_{1}^{\prime} \varphi\left(b, \lambda_{2}\right)-\gamma_{2}^{\prime} \varphi^{\prime}\left(b, \lambda_{2}\right)\right)=0 .
\end{aligned}
$$

We define the solutions

$$
\varphi(x, \lambda)=\left\{\begin{array}{ll}
\varphi_{1}(x, \lambda), & x \in\left[a, \delta_{1}\right), \\
\varphi_{2}(x, \lambda), & x \in\left(\delta_{1}, \delta_{2}\right), \\
\varphi_{3}(x, \lambda), & x \in\left(\delta_{2}, b\right],
\end{array} \quad \psi(x, \lambda)= \begin{cases}\psi_{1}(x, \lambda), & x \in\left[a, \delta_{1}\right), \\
\psi_{2}(x, \lambda), & x \in\left(\delta_{1}, \delta_{2}\right), \\
\psi_{3}(x, \lambda), & x \in\left(\delta_{2}, b\right]\end{cases}\right.
$$

of equation (1) by the initial conditions

$$
\begin{aligned}
& \varphi_{1}(a, \lambda)=\lambda \theta_{2}^{\prime}-\theta_{2}, \quad \varphi_{1}^{\prime}(a, \lambda)=\lambda \theta_{1}^{\prime}-\theta_{1}, \\
& \varphi_{2}\left(\delta_{1}, \lambda\right)=\theta_{3} \varphi_{1}\left(\delta_{1}, \lambda\right)+\gamma_{3} \varphi_{1}^{\prime}\left(\delta_{1}, \lambda\right), \quad \varphi_{2}^{\prime}\left(\delta_{1}, \lambda\right)=\theta_{4} \varphi_{1}\left(\delta_{1}, \lambda\right)+\gamma_{4} \varphi_{1}^{\prime}\left(\delta_{1}, \lambda\right), \\
& \varphi_{3}\left(\delta_{2}, \lambda\right)=\theta_{5} \varphi_{2}\left(\delta_{2}, \lambda\right)+\gamma_{5} \varphi_{2}^{\prime}\left(\delta_{2}, \lambda\right), \quad \varphi_{3}^{\prime}\left(\delta_{2}, \lambda\right)=\theta_{6} \varphi_{2}\left(\delta_{2}, \lambda\right)+\gamma_{6} \varphi_{2}^{\prime}\left(\delta_{2}, \lambda\right),
\end{aligned}
$$

and similarly,

$$
\begin{array}{ll}
\psi_{3}(b, \lambda)=\lambda \gamma_{2}^{\prime}+\gamma_{2}, \quad \psi_{3}^{\prime}(b, \lambda)=\lambda \gamma_{1}^{\prime}+\gamma_{1}, \\
\psi_{2}\left(\delta_{2}, \lambda\right)=\frac{\gamma_{6} \psi_{3}\left(\delta_{2}, \lambda\right)-\gamma_{5} \psi_{3}^{\prime}\left(\delta_{2}, \lambda\right)}{\alpha_{2}}, & \psi_{2}^{\prime}\left(\delta_{2}, \lambda\right)=\frac{\theta_{6} \psi_{3}\left(\delta_{2}, \lambda\right)-\theta_{5} \psi_{3}^{\prime}\left(\delta_{2}, \lambda\right)}{-\alpha_{2}}, \\
\psi_{1}\left(\delta_{1}, \lambda\right)=\frac{\gamma_{4} \psi_{2}\left(\delta_{1}, \lambda\right)-\gamma_{3} \psi_{2}^{\prime}\left(\delta_{1}, \lambda\right)}{\alpha_{1}}, & \psi_{1}^{\prime}\left(\delta_{1}, \lambda\right)=\frac{\theta_{4} \psi_{2}\left(\delta_{1}, \lambda\right)-\theta_{3} \psi_{2}^{\prime}\left(\delta_{1}, \lambda\right)}{-\alpha_{1}},
\end{array}
$$

respectively.

These solutions are entire functions of $\lambda$ for each fixed $x \in[a, b]$ and satisfy the relation

$$
\psi\left(x, \lambda_{n}\right)=\kappa_{n} \varphi\left(x, \lambda_{n}\right)
$$

for each eigenvalue $\lambda_{n}$, where

$$
\kappa_{n}=\frac{\theta_{2}^{\prime} \psi^{\prime}\left(a, \lambda_{n}\right)-\theta_{1}^{\prime} \psi\left(a, \lambda_{n}\right)}{\beta_{1}} .
$$


Lemma 2 Let $\lambda=k^{2}, k=\sigma+i \omega$.

Then the following integral equations and also asymptotic behaviors hold for $v=0,1$ :

$$
\begin{aligned}
& \frac{d^{\nu}}{d x^{\nu}} \varphi_{1}(x, \lambda)=\left(\lambda \theta_{2}^{\prime}-\theta_{2}\right) \frac{d^{\nu}}{d x^{\nu}} \cos k \rho_{1}(x-a) \\
& +\frac{1}{k \rho_{1}}\left(\lambda \theta_{1}^{\prime}-\theta_{1}\right) \frac{d^{v}}{d x^{v}} \sin k \rho_{1}(x-a) \\
& +\frac{\rho_{1}}{k} \int_{a}^{x} \frac{d^{v}}{d x^{\nu}} \sin k \rho_{1}(x-t) q(t) \varphi_{1}(t, \lambda) d t \\
& =\left(\lambda \theta_{2}^{\prime}-\theta_{2}\right) \frac{d^{\nu}}{d x^{\nu}} \cos k \rho_{1}(x-a) \\
& +\frac{1}{k \rho_{1}}\left(\lambda \theta_{1}^{\prime}-\theta_{1}\right) \frac{d^{\nu}}{d x^{\nu}} \sin k \rho_{1}(x-a)+O\left(|k|^{\nu+1} e^{|\operatorname{Im} k|(x-a) \rho_{1}}\right), \\
& \frac{d^{\nu}}{d x^{\nu}} \varphi_{2}(x, \lambda)=\left(\theta_{3} \varphi_{1}\left(\delta_{1}, \lambda\right)+\gamma_{3} \varphi_{1}^{\prime}\left(\delta_{1}, \lambda\right)\right) \frac{d^{\nu}}{d x^{\nu}} \cos k \rho_{2}\left(x-\delta_{1}\right) \\
& +\frac{1}{k \rho_{2}}\left(\theta_{4} \varphi_{1}\left(\delta_{1}, \lambda\right)+\gamma_{4} \varphi_{1}^{\prime}\left(\delta_{1}, \lambda\right)\right) \frac{d^{\nu}}{d x^{\nu}} \sin k \rho_{2}\left(x-\delta_{1}\right) \\
& +\frac{\rho_{2}}{k} \int_{\delta_{1}}^{x} \frac{d^{v}}{d x^{\nu}} \sin k \rho_{2}(x-t) q(t) \varphi_{2}(t, \lambda) d t \\
& =-\left(\lambda \theta_{2}^{\prime}-\theta_{2}\right) \gamma_{3} k \rho_{1} \sin k \rho_{1}\left(\delta_{1}-a\right) \frac{d^{\nu}}{d x^{\nu}} \cos k \rho_{2}\left(x-\delta_{1}\right) \\
& +O\left(|k|^{v+2} e^{|\operatorname{Im} k|\left(\left(\delta_{1}-a\right) \rho_{1}+\left(x-\delta_{1}\right) \rho_{2}\right)}\right), \\
& \frac{d^{\nu}}{d x^{\nu}} \varphi_{3}(x, \lambda)=\left(\theta_{5} \varphi_{2}\left(\delta_{2}, \lambda\right)+\gamma_{5} \varphi_{2}^{\prime}\left(\delta_{2}, \lambda\right)\right) \frac{d^{\nu}}{d x^{\nu}} \cos k \rho_{3}\left(x-\delta_{2}\right) \\
& +\frac{1}{k \rho_{3}}\left(\theta_{5} \varphi_{2}\left(\delta_{2}, \lambda\right)+\gamma_{6} \varphi_{2}^{\prime}\left(\delta_{2}, \lambda\right)\right) \frac{d^{\nu}}{d x^{\nu}} \sin k \rho_{3}\left(x-\delta_{2}\right) \\
& +\frac{\rho_{3}}{k} \int_{\delta_{2}}^{x} \frac{d^{\nu}}{d x^{\nu}} \sin k \rho_{3}(x-t) q(t) \varphi_{3}(t, \lambda) d t \\
& =\left(\lambda \theta_{2}^{\prime}-\theta_{2}\right) \gamma_{3} \gamma_{5} k^{2} \rho_{1} \rho_{2} \sin k \rho_{1}\left(\delta_{1}-a\right) \sin k \rho_{2}\left(\delta_{2}-\delta_{1}\right) \frac{d^{\nu}}{d x^{\nu}} \cos k \rho_{3}\left(x-\delta_{2}\right) \\
& +O\left(|k|^{v+3} e^{|\operatorname{Im} k|\left(\left(\delta_{1}-a\right) \rho_{1}+\left(\delta_{2}-\delta_{1}\right) \rho_{2}+\left(x-\delta_{2}\right) \rho_{3}\right)}\right) .
\end{aligned}
$$

Lemma 3 Let $\lambda=k^{2}, k=\sigma+i \omega$.

Then the following integral equations and also asymptotic behaviors hold for $v=0,1$ :

$$
\begin{aligned}
\frac{d^{\nu}}{d x^{\nu}} \psi_{3}(x, \lambda)= & \left(\lambda \gamma_{2}^{\prime}+\gamma_{2}\right) \frac{d^{\nu}}{d x^{\nu}} \cos k \rho_{3}(x-b) \\
& +\frac{1}{k \rho_{3}}\left(\lambda \gamma_{1}^{\prime}+\gamma_{1}\right) \frac{d^{\nu}}{d x^{\nu}} \sin k \rho_{3}(x-b) \\
& -\frac{\rho_{3}}{k} \int_{x}^{b} \frac{d^{\nu}}{d x^{\nu}} \sin k \rho_{3}(x-t) q(t) \psi_{3}(t, \lambda) d t \\
= & \left(\lambda \gamma_{2}^{\prime}+\gamma_{2}\right) \frac{d^{\nu}}{d x^{\nu}} \cos k \rho_{3}(x-b) \\
& +\frac{1}{k \rho_{3}}\left(\lambda \gamma_{1}^{\prime}+\gamma_{1}\right) \frac{d^{\nu}}{d x^{\nu}} \sin k \rho_{3}(x-b)+O\left(|k|^{\nu+1} e^{|\operatorname{Im} k|(x-b) \rho_{3}}\right),
\end{aligned}
$$




$$
\begin{aligned}
& \frac{d^{\nu}}{d x^{\nu}} \psi_{2}(x, \lambda)=\frac{\gamma_{6} \psi_{3}\left(\delta_{2}, \lambda\right)-\gamma_{5} \psi_{3}^{\prime}\left(\delta_{2}, \lambda\right)}{\alpha_{2}} \frac{d^{\nu}}{d x^{\nu}} \cos k \rho_{2}\left(x-\delta_{2}\right) \\
& +\frac{1}{k \rho_{2}} \frac{\theta_{6} \psi_{3}\left(\delta_{2}, \lambda\right)-\theta_{5} \psi_{3}^{\prime}\left(\delta_{2}, \lambda\right)}{-\alpha_{2}} \frac{d^{v}}{d x^{\nu}} \sin k \rho_{2}\left(x-\delta_{2}\right) \\
& -\frac{\rho_{2}}{k} \int_{x}^{\delta_{2}} \frac{d^{\nu}}{d x^{\nu}} \sin k \rho_{2}(x-t) q(t) \psi_{2}(t, \lambda) d t \\
& =\left(\frac{\lambda \gamma_{2}^{\prime}+\gamma_{2}}{\alpha_{2}}\right)\left(k \rho_{3} \gamma_{5} \sin k \rho_{3}\left(\delta_{2}-b\right) \frac{d^{\nu}}{d x^{\nu}} \cos k \rho_{2}\left(x-\delta_{2}\right)\right) \\
& +O\left(|k|^{v+2} e^{|\operatorname{Im} k|\left(\left(\delta_{2}-b\right) \rho_{3}+\left(x-\delta_{2}\right) \rho_{2}\right)}\right), \\
& \frac{d^{\nu}}{d x^{\nu}} \psi_{1}(x, \lambda)=\frac{\gamma_{4} \psi_{2}\left(\delta_{1}, \lambda\right)-\gamma_{3} \psi_{2}^{\prime}\left(\delta_{1}, \lambda\right)}{\alpha_{1}} \frac{d^{\nu}}{d x^{\nu}} \cos k \rho_{1}\left(x-\delta_{1}\right) \\
& +\frac{1}{k \rho_{1}} \frac{\theta_{4} \psi_{2}\left(\delta_{1}, \lambda\right)-\theta_{3} \psi_{2}^{\prime}\left(\delta_{1}, \lambda\right)}{-\alpha_{1}} \frac{d^{\nu}}{d x^{\nu}} \sin k \rho_{1}\left(x-\delta_{1}\right) \\
& -\frac{\rho_{1}}{k} \int_{x}^{\delta_{1}} \frac{d^{v}}{d x^{v}} \sin k \rho_{1}(x-t) q(t) \psi_{1}(t, \lambda) d t \\
& =\left(\frac{\lambda \gamma_{2}^{\prime}+\gamma_{2}}{\alpha_{2} \alpha_{1}}\right) \\
& \times\left(k^{2} \rho_{2} \rho_{3} \gamma_{3} \gamma_{5} \sin k \rho_{3}\left(\delta_{2}-b\right) \sin k \rho_{2}\left(\delta_{1}-\delta_{2}\right) \frac{d^{v}}{d x^{\nu}} \cos k \rho_{1}\left(x-\delta_{1}\right)\right) \\
& +O\left(|k|^{v+3} e^{|\operatorname{Im} k|\left(\left(\delta_{2}-b\right) \rho_{3}+\left(\delta_{1}-\delta_{2}\right) \rho_{2}+\left(x-\delta_{1}\right) \rho_{1}\right)}\right) .
\end{aligned}
$$

The function $\Delta(\lambda)$ is called the characteristic function, and numbers $\left\{\mu_{n}\right\}_{n \geq 1}$ are called the normalizing constants of problem (1)-(7) such that

$$
\begin{aligned}
\Delta(\lambda)= & \lambda\left(\gamma_{1}^{\prime} \varphi(b, \lambda)-\gamma_{2}^{\prime} \varphi^{\prime}(b, \lambda)\right)+\left(\gamma_{1} \varphi(b, \lambda)-\gamma_{2}^{\prime} \varphi^{\prime}(b, \lambda)\right), \\
\mu_{n}:= & \alpha_{1} \alpha_{2} \rho_{1} \int_{a}^{\delta_{1}} \varphi^{2}\left(x, \lambda_{n}\right) d x+\alpha_{2} \rho_{2} \int_{\delta_{1}}^{\delta_{2}} \varphi^{2}\left(x, \lambda_{n}\right) d x+\rho_{3} \int_{\delta_{2}}^{b} \varphi^{2}\left(x, \lambda_{n}\right) d x \\
& +\frac{\alpha_{1} \alpha_{2}}{\beta_{1}}\left(\theta_{1}^{\prime} \varphi\left(a, \lambda_{n}\right)-\theta_{2}^{\prime} \varphi^{\prime}\left(a, \lambda_{n}\right)\right)^{2}+\frac{1}{\beta_{2}}\left(\gamma_{1}^{\prime} \varphi\left(b, \lambda_{n}\right)-\gamma_{2}^{\prime} \varphi^{\prime}\left(b, \lambda_{n}\right)\right)^{2} .
\end{aligned}
$$

Lemma 4 The following equality holds for each eigenvalue $\lambda_{n}$

$$
\alpha_{1} \alpha_{2} \dot{\Delta}\left(\lambda_{n}\right)=\kappa_{n} \mu_{n}
$$

Proof Since

$$
-\rho(x) \psi^{\prime \prime}(x, \lambda)+q(x) \psi(x, \lambda)=\lambda \psi(x, \lambda), \quad-\rho(x) \varphi^{\prime \prime}\left(x, \lambda_{n}\right)+q(x) \varphi\left(x, \lambda_{n}\right)=\lambda \varphi\left(x, \lambda_{n}\right),
$$

we get

$$
\begin{aligned}
\varphi^{\prime}\left(x, \lambda_{n}\right) \psi(x, \lambda)-\psi^{\prime}(x, \lambda) \varphi\left(x, \lambda_{n}\right)\left(\left.\right|_{a} ^{\delta_{1}}+\left.\right|_{\delta_{1}} ^{\delta_{2}}+\left.\right|_{\delta_{2}} ^{b}\right) \\
=\left(\lambda-\lambda_{n}\right) \rho_{1}^{2} \int_{a}^{\delta_{1}} \psi(x, \lambda) \varphi\left(x, \lambda_{n}\right) d x \\
\quad+\left(\lambda-\lambda_{n}\right) \rho_{2}^{2} \int_{\delta_{1}}^{\delta_{2}} \psi(x, \lambda) \varphi\left(x, \lambda_{n}\right) d x+\left(\lambda-\lambda_{n}\right) \rho_{3}^{2} \int_{\delta_{2}}^{b} \psi(x, \lambda) \varphi\left(x, \lambda_{n}\right) d x .
\end{aligned}
$$


After that, add and subtract $\Delta(\lambda)$ on the left-hand side of the last equality, and by using conditions (2)-(7), we obtain

$$
\begin{aligned}
\Delta(\lambda) & +\left(\lambda-\lambda_{n}\right)\left(\theta_{1}^{\prime} \psi(a, \lambda)-\theta_{2}^{\prime} \psi^{\prime}(a, \lambda)\right)-\left(\lambda-\lambda_{n}\right)\left(\gamma_{1}^{\prime} \varphi\left(b, \lambda_{n}\right)-\gamma_{2}^{\prime} \varphi^{\prime}\left(b, \lambda_{n}\right)\right) \\
& +\left(1-\alpha_{1}\right)\left(\psi\left(\delta_{1}^{-}, \lambda\right) \varphi^{\prime}\left(\delta_{1}^{-}, \lambda_{n}\right)-\varphi\left(\delta_{1}^{-}, \lambda_{n}\right) \psi^{\prime}\left(\delta_{1}^{-}, \lambda\right)\right) \\
& +\left(1-\alpha_{2}\right)\left(\psi\left(\delta_{2}^{-}, \lambda\right) \varphi^{\prime}\left(\delta_{2}^{-}, \lambda_{n}\right)-\varphi\left(\delta_{2}^{-}, \lambda_{n}\right) \psi^{\prime}\left(\delta_{2}^{-}, \lambda\right)\right) \\
= & \left(\lambda-\lambda_{n}\right) \rho_{1}^{2} \int_{a}^{\delta_{1}} \psi(x, \lambda) \varphi\left(x, \lambda_{n}\right) d x+\left(\lambda-\lambda_{n}\right) \rho_{2}^{2} \int_{\delta_{1}}^{\delta_{2}} \psi(x, \lambda) \varphi\left(x, \lambda_{n}\right) d x \\
& +\left(\lambda-\lambda_{n}\right) \rho_{3}^{2} \int_{\delta_{2}}^{b} \psi(x, \lambda) \varphi\left(x, \lambda_{n}\right) d x,
\end{aligned}
$$

or

$$
\begin{aligned}
\frac{\alpha_{1} \alpha_{2} \Delta(\lambda)}{\lambda-\lambda_{n}}= & \alpha_{1} \alpha_{2} \rho_{1}^{2} \int_{a}^{\delta_{1}} \psi(x, \lambda) \varphi\left(x, \lambda_{n}\right) d x+\alpha_{2} \rho_{2}^{2} \int_{\delta_{1}}^{\delta_{2}} \psi(x, \lambda) \varphi\left(x, \lambda_{n}\right) d x \\
& +\rho_{3}^{2} \int_{\delta_{2}}^{b} \psi(x, \lambda) \varphi\left(x, \lambda_{n}\right) d x \\
& +\frac{\alpha_{1} \alpha_{2}}{\beta_{1}}\left(\theta_{1}^{\prime} \psi(a, \lambda)-\theta_{2}^{\prime} \psi^{\prime}(a, \lambda)\right)\left(\theta_{1}^{\prime} \varphi\left(a, \lambda_{n}\right)-\theta_{2}^{\prime} \varphi^{\prime}\left(a, \lambda_{n}\right)\right) \\
& +\frac{1}{\beta_{2}}\left(\gamma_{1}^{\prime} \varphi\left(b, \lambda_{n}\right)-\gamma_{2}^{\prime} \varphi^{\prime}\left(b, \lambda_{n}\right)\right)\left(\gamma_{1}^{\prime} \psi(b, \lambda)-\gamma_{2}^{\prime} \psi^{\prime}(b, \lambda)\right) .
\end{aligned}
$$

For $\lambda \rightarrow \lambda_{n}, \alpha_{1} \alpha_{2} \dot{\Delta}\left(\lambda_{n}\right)=\kappa_{n} \mu_{n}$ is obtained by using the equality

$$
\psi\left(x, \lambda_{n}\right)=\kappa_{n} \varphi\left(x, \lambda_{n}\right)
$$

and (12).

Corollary 2 The eigenvalues of problem L are simple.

Lemma 5 [36] Let $\left\{\alpha_{i}\right\}_{i=1}^{p}$ be the set of real numbers satisfying the inequalities $\alpha_{0}>\alpha_{0}>$ $\cdots>\alpha_{p-1}>0$, and let $\left\{a_{i}\right\}_{i=1}^{p}$ be the set of complex numbers. If $a_{p} \neq 0$, then the roots of the equation

$$
e^{\alpha_{0} \lambda}+a_{1} e^{\alpha_{1} \lambda}+\cdots+a_{p-1} e^{\alpha_{0} \lambda}+a_{p}=0
$$

have the form

$$
\lambda_{n}=\frac{2 \pi n i}{\alpha_{0}}+\Psi(n) \quad(n=0, \pm 1, \ldots)
$$

where $\Psi(n)$ is a bounded sequence.

Now, from Lemma 2 and (11), we can write

$$
\Delta(\lambda)-\Delta_{0}(\lambda)=O\left(k^{6} e^{|\operatorname{Im} k|\left(\left(\delta_{1}-a\right) \rho_{1}+\left(\delta_{2}-\delta_{1}\right) \rho_{2}+\left(b-\delta_{2}\right) \rho_{3}\right)}\right),
$$

where $\Delta_{0}(\lambda)=k^{7} \theta_{2}^{\prime} \gamma_{2}^{\prime} \gamma_{3} \gamma_{5} \rho_{1} \rho_{2} \rho_{3} \sin k \rho_{1}\left(\delta_{1}-a\right) \sin k \rho_{2}\left(\delta_{2}-\delta_{1}\right) \sin k \rho_{3}\left(b-\delta_{2}\right)$. 
We can see that non-zero roots, namely $\lambda_{n}^{0}$ of the equation $\Delta_{0}(\lambda)=0$, are real and analytically simple.

Furthermore, it can be proved by using Lemma 5 that

$$
\sqrt{\lambda_{n}^{0}}=\frac{n \pi}{\left(\delta_{1}-a\right) \rho_{1}+\left(\delta_{2}-\delta_{1}\right) \rho_{2}+\left(b-\delta_{2}\right) \rho_{3}}+\Psi_{n}, \quad \sup _{n}\left|\Psi_{n}\right|<\infty .
$$

Theorem 2 The eigenvalues $\left\{\lambda_{n}\right\}_{n \geq 0}$ have the following asymptotic behavior for sufficiently large n:

$$
\sqrt{\lambda_{n}}=\sqrt{\lambda_{n-4}^{0}}+o(1)
$$

Proof Denote

$$
G_{n}:=\left\{\lambda: k^{2}=\lambda,|k| \leq\left|k_{n}^{0}\right|+\delta\right\}
$$

where $k_{n}^{0}=\sqrt{\lambda_{n}^{0}}$ and $\delta$ is a sufficiently small number. The relations

$$
\left|\Delta_{0}(\lambda)\right| \geq C_{\delta}|k|^{7} e^{|\operatorname{Im} k|\left(\left(\delta_{1}-a\right) \rho_{1}+\left(\delta_{2}-\delta_{1}\right) \rho_{2}+\left(b-\delta_{2}\right) \rho_{3}\right)}
$$

and

$$
\Delta(\lambda)-\Delta_{0}(\lambda)=O\left(k^{6} e^{|\operatorname{Im} k|\left(\left(\delta_{1}-a\right) \rho_{1}+\left(\delta_{2}-\delta_{1}\right) \rho_{2}+\left(b-\delta_{2}\right) \rho_{3}\right)}\right)
$$

are valid for $\lambda \in \partial G_{n}$.

Then, by Rouche's theorem that the number of zeros of $\Delta_{0}(\lambda)$ coincides with the number of zeros of $\Delta(\lambda)$ in $G_{n}$, namely $n+4$ zeros, $\lambda_{0}, \lambda_{1}, \lambda_{2}, \ldots, \lambda_{n+3}$. In the annulus, between $G_{n}$ and $G_{n+1}, \Delta(\lambda)$ has accurately one positive zero, namely $k_{n}^{2}: k_{n}=\sqrt{\lambda_{n}^{0}}+\delta_{n}$, for $n \geq 1$. So, it follows that $\lambda_{n+4}=k_{n}^{2}$. Applying to Rouche's theorem in $\eta_{\varepsilon}=\left\{k:\left|k-k_{n}^{0}\right|<\varepsilon\right\}$ for sufficiently small $\varepsilon$ and sufficiently large $n$, we get $\delta_{n}=o(1)$. Finally, we obtain the asymptotic formula

$$
\sqrt{\lambda_{n}}=\sqrt{\lambda_{n-4}^{0}}+o(1)
$$

Denote

$$
\Delta_{i}(\lambda):=W\left(\varphi_{i}, \psi_{i}, x\right):=\varphi_{i} \psi_{i}^{\prime}-\varphi_{i}^{\prime} \psi_{i}, \quad x \in \Omega_{i}(i=\overline{1,3}),
$$

which are independent of $x \in \Omega_{i}$ and are entire functions such that $\Omega_{1}=\left[a, \delta_{1}\right), \Omega_{2}=$ $\left(\delta_{1}, \delta_{2}\right), \Omega_{3}=\left(\delta_{2}, b\right]$.

It can be easily seen that

$$
\Delta(\lambda):=\Delta_{3}(\lambda)=\alpha_{2} \Delta_{2}(\lambda)=\alpha_{1} \alpha_{2} \Delta_{1}(\lambda) .
$$

Example Let $q=0, a=0, b=\pi, \delta_{1}=\frac{\pi}{4}, \delta_{2}=\frac{\pi}{2}, \theta_{3}=\gamma_{4}=0, \gamma_{3}=1, \theta_{4}=-1, \theta_{5}=\gamma_{6}=0$, $\gamma_{5}=1, \theta_{6}=-1, \gamma_{2}^{\prime}=1, \gamma_{6}=-1, \gamma_{1}^{\prime}=\gamma_{2}=0, \theta_{2}^{\prime}=1 \theta_{1}=-1, \theta_{1}^{\prime}=\theta_{2}=0$. 
Since

$$
\begin{aligned}
\Delta(\lambda)= & \rho_{1} \rho_{2} \rho_{3} k^{7} \sin k \rho_{1} \delta_{1} \sin k \rho_{2}\left(\delta_{2}-\delta_{1}\right) \sin k \rho_{3}\left(\pi-\delta_{2}\right) \\
& +O\left(k^{6} e^{|\operatorname{Im} k|\left(\delta_{1} \rho_{1}+\left(\delta_{2}-\delta_{1}\right) \rho_{2}+\left(\pi-\delta_{2}\right) \rho_{3}\right)}\right),
\end{aligned}
$$

the eigenvalues of the boundary value problem (1)-(7) satisfy the following asymptotic formulae:

$$
\begin{aligned}
& \sqrt{\lambda_{n_{1}}}=k_{n_{1}}=\frac{4(n-4)}{\rho_{1}}+\varepsilon_{n}, \quad \sqrt{\lambda_{n_{2}}}=k_{n_{2}}=\frac{4(n-4)}{\rho_{2}}+\varepsilon_{n}, \\
& \sqrt{\lambda_{n_{3}}}=k_{n_{3}}=\frac{2(n-4)}{\rho_{3}}+\varepsilon_{n},
\end{aligned}
$$

where $\varepsilon_{n}=O\left(n^{-1}\right)$.

\section{Inverse problems}

In this section, we study the inverse problems for the reconstruction of the boundary value problem (1)-(7) by Weyl function and spectral data.

We consider the boundary value problem $\tilde{L}$ with the same form of $L$ but with different coefficients $\tilde{q}(x), \tilde{\theta}_{i}, \tilde{\gamma}_{i}, \tilde{\delta}_{j}, \tilde{\theta}_{j}^{\prime}, \tilde{\gamma}_{j}^{\prime}, i=1-6, j=1,2$.

If a certain symbol $\alpha$ denotes an object related to $L$, then the symbol $\tilde{\alpha}$ denotes the corresponding object related to $\tilde{L}$.

The Weyl function Let $\Phi(x, \lambda)$ be a solution of equation (1), which satisfies the condition $\left(\lambda \theta_{1}^{\prime}-\theta_{1}\right) \Phi(a, \lambda)-\left(\lambda \theta_{2}^{\prime}-\theta_{2}\right) \Phi(a, \lambda)=1$ and transmissions (4)-(7).

Assume that the function $\chi(x, \lambda)$ is the solution of equation (1) that satisfies the conditions $\chi(a, \lambda)=\beta_{1}^{-1} \theta_{2}^{\prime}, \chi^{\prime}(a, \lambda)=\beta_{1}^{-1} \theta_{1}^{\prime}$ and the transmission conditions (4)-(7).

Since $W[\chi, \varphi]=1$, the functions $\chi$ and $\varphi$ are linearly independent. Therefore, the function $\psi(x, \lambda)$ can be represented by

$$
\psi(x, \lambda)=\frac{\theta_{2}^{\prime} \psi^{\prime}(a, \lambda)-\theta_{1}^{\prime} \psi(a, \lambda)}{\beta_{1}} \varphi(x, \lambda)+\Delta(\lambda) \chi(x, \lambda)
$$

or

$$
\Phi(x, \lambda)=\frac{\psi(x, \lambda)}{\Delta(\lambda)}=\chi(x, \lambda)+\frac{\theta_{2}^{\prime} \psi^{\prime}(a, \lambda)-\theta_{1}^{\prime} \psi(a, \lambda)}{\beta_{1} \Delta(\lambda)} \varphi(x, \lambda)
$$

that is called the Weyl solution, and

$$
\frac{\theta_{2}^{\prime} \psi^{\prime}(a, \lambda)-\theta_{1}^{\prime} \psi(a, \lambda)}{\beta_{1} \Delta(\lambda)}=M(\lambda)
$$

is called the Weyl function.

Theorem 3 If $M(\lambda)=\tilde{M}(\lambda)$, then $L=\tilde{L}$, i.e., $q(x)=\tilde{q}(x)$, a.e. and $\theta_{i}=\tilde{\theta}_{i}, \gamma_{i}=\tilde{\gamma}_{i}, i=1-6$, $\delta_{j}=\tilde{\delta}_{j}, \theta_{j}^{\prime}=\tilde{\theta}_{j}^{\prime}, \gamma_{j}^{\prime}=\tilde{\gamma}_{j}^{\prime}, j=1,2$. 
Proof We introduce a matrix $P(x, \lambda)=\left[P_{k j}(x, \lambda)\right]_{k, j=1,2}$ by the formula

$$
P(x, \lambda)\left(\begin{array}{cc}
\tilde{\varphi} & \tilde{\Phi} \\
\tilde{\varphi}^{\prime} & \tilde{\Phi}^{\prime}
\end{array}\right)=\left(\begin{array}{cc}
\varphi & \Phi \\
\varphi^{\prime} & \Phi^{\prime}
\end{array}\right)
$$

or

$$
\left(\begin{array}{ll}
P_{11}(x, \lambda) & P_{12}(x, \lambda) \\
P_{21}(x, \lambda) & P_{22}(x, \lambda)
\end{array}\right)=\left(\begin{array}{cc}
-\varphi \tilde{\Phi}^{\prime}+\Phi \tilde{\varphi}^{\prime} & \varphi \tilde{\Phi}-\Phi \tilde{\varphi} \\
-\varphi^{\prime} \tilde{\Phi}^{\prime}+\tilde{\varphi}^{\prime} \Phi^{\prime} & \varphi^{\prime} \tilde{\Phi}-\tilde{\varphi} \Phi^{\prime}
\end{array}\right)
$$

where $\Phi(x, \lambda)=\frac{\psi(x, \lambda)}{\Delta(\lambda)}$.

$$
\begin{aligned}
& P_{11}(x, \lambda)=\chi(x, \lambda) \tilde{\varphi}^{\prime}(x, \lambda)-\varphi(x, \lambda) \tilde{\chi}^{\prime}(x, \lambda)+(M(\lambda)-\tilde{M}(\lambda)) \varphi(x, \lambda) \tilde{\varphi}^{\prime}(x, \lambda), \\
& P_{12}(x, \lambda)=\varphi(x, \lambda) \tilde{\chi}(x, \lambda)-\chi(x, \lambda) \tilde{\varphi}(x, \lambda)+(\tilde{M}(\lambda)-M(\lambda)) \varphi(x, \lambda) \tilde{\varphi}(x, \lambda) .
\end{aligned}
$$

Thus, if $M(\lambda) \equiv \tilde{M}(\lambda)$, then the functions $P_{11}(x, \lambda)$ and $P_{12}(x, \lambda)$ are entire in $\lambda$ for each fixed $x$.

Denote $G_{w}=\left\{\lambda: \lambda=k^{2},\left|k-k_{\delta}\right|>w, \delta=1,2, \ldots\right\}$ and $\tilde{G}_{w}=\left\{\lambda: \lambda=k^{2},\left|k-\tilde{k}_{\delta}\right|>w, \delta=\right.$ $1,2, \ldots\}$, where $w$ is sufficiently small number, $k_{\delta}$ and $\tilde{k}_{\delta}$ are square roots of the eigenvalues of the problem $L$ and $\tilde{L}$, respectively. It is easily shown that

$$
\begin{aligned}
& \Phi_{i}^{(v)}(x, \lambda) \leq C_{w}|k|^{\nu-(2+i)} e^{-|\operatorname{Im} \sqrt{\lambda}|\left(\left(\delta_{1}-a\right) \rho_{1}+\left(\delta_{2}-\delta_{1}\right) \rho_{2}+\left(b-\delta_{2}\right) \rho_{3}\right)}, \\
& x \in \Omega_{i}(i=1,2,3), v=0,1
\end{aligned}
$$

are valid for sufficiently large $|\lambda|$, where $\Omega_{1}=\left[a, \delta_{1}\right), \Omega_{2}=\left(\delta_{1}, \delta_{2}\right)$ and $\Omega_{3}=\left(\delta_{2}, b\right]$. Hence, Lemma 2 and (18) yield that

$$
\left|P_{11}(x, \lambda)\right| \leq C_{w}, \quad\left|P_{12}(x, \lambda)\right| \leq C_{w}|k|^{-1} \quad \text { for } \lambda \in \Omega \text { and for } \lambda \in G_{w} \cap \tilde{G}_{w} .
$$

According to (19), and Liouville's theorem, $P_{11}(x, \lambda)=C(x)$ and $P_{12}(x, \lambda) \equiv 0$ for $x \in$ $[a, b] \backslash\left\{\delta_{1}, \delta_{2}, \tilde{\delta}_{1}, \tilde{\delta}_{2}\right\}$. By virtue of (17), we get

$$
\varphi(x, \lambda)=C(x) \tilde{\varphi}(x, \lambda), \quad \Phi(x, \lambda)=C(x) \tilde{\Phi}(x, \lambda) .
$$

It is obvious that

$$
\begin{aligned}
W[\Phi(x, \lambda), \varphi(x, \lambda)] & =\Phi(a, \lambda)\left(\theta_{1}^{\prime} \lambda-\theta_{1}\right)-\Phi^{\prime}(a, \lambda)\left(\theta_{2}^{\prime} \lambda-\theta_{2}\right) \\
& =\frac{\psi(a, \lambda)\left(\theta_{1}^{\prime} \lambda-\theta_{1}\right)-\psi^{\prime}(a, \lambda)\left(\theta_{2}^{\prime} \lambda-\theta_{2}\right)}{\Delta(\lambda)} \equiv 1,
\end{aligned}
$$

and similarly, $W[\tilde{\Phi}(x, \lambda), \tilde{\varphi}(x, \lambda)] \equiv 1$. Thus, we have $C^{2}(x) \equiv 1$. 
Otherwise, the following asymptotic expressions hold

$$
\begin{aligned}
& \varphi_{1}(x, \lambda)=\tilde{\varphi}_{1}(x, \lambda)=\frac{\lambda}{2} e^{-i k(x-a) \rho_{1}}(1+o(1)) \quad \text { for } x<\delta_{1} \text { and } x<\tilde{\delta}_{1}, \\
& \varphi_{2}(x, \lambda)=\tilde{\varphi}_{2}(x, \lambda)=-\frac{\lambda^{3 \backslash 2}}{2} e^{-i k\left(\left(\delta_{1}-a\right) \rho_{1}+\left(x-\delta_{1}\right) \rho_{2}\right)}(1+o(1)) \\
& \text { for } \delta_{1}<x<\delta_{2} \text { and } \tilde{\delta}_{1}<x<\tilde{\delta}_{2}, \\
& \varphi_{3}(x, \lambda)=\tilde{\varphi}_{3}(x, \lambda)=\frac{\lambda^{2}}{2} e^{-i k\left(\left(\delta_{1}-a\right) \rho_{1}+\left(\delta_{2}-\delta_{1}\right) \rho_{2}+\left(x-\delta_{2}\right) \rho_{3}\right)}(1+o(1)) \\
& \text { for } \delta_{2}<x \text { and } \tilde{\delta}_{2}<x
\end{aligned}
$$

Without loss of generality, we assume that $\delta_{1}<\tilde{\delta}_{1}$ and $\delta_{2}<\tilde{\delta}_{2}$. From (20)-(21), we get $C(x) \equiv 1$ for $x \in\left[a, \delta_{1}\right) \cup\left(\tilde{\delta}_{1}, \delta_{2}\right) \cup\left(\tilde{\delta}_{2}, b\right]$ and also

$$
\begin{aligned}
& \frac{1}{\lambda^{1 / 2}}(1+o(1)) C(x)=-e^{\left(x-\delta_{1}\right) \rho_{2}}(1+o(1)) \quad \text { for } x \in\left(\delta_{1}, \tilde{\delta}_{1}\right), \\
& \frac{1}{\lambda^{1 / 2}} e^{\left(x-\delta_{1}\right) \rho_{2}}(1+o(1)) C(x)=-e^{\left(\delta_{2}-\delta_{1}\right) \rho_{2}+\left(x-\delta_{2}\right) \rho_{3}}(1+o(1)) \quad \text { for } x \in\left(\delta_{2}, \tilde{\delta}_{2}\right) .
\end{aligned}
$$

As $|\lambda| \rightarrow \infty$ in (22), we contradict $C^{2}(x) \equiv 1$. Therefore, $\delta_{1}=\tilde{\delta}_{1}, \delta_{2}=\tilde{\delta}_{2}$. Thus, $\varphi(x, \lambda) \equiv$ $\tilde{\varphi}(x, \lambda), \Phi(x, \lambda) \equiv \tilde{\Phi}(x, \lambda)$ and $\frac{\psi^{\prime}(x, \lambda)}{\psi(x, \lambda)} \equiv \frac{\tilde{\psi}^{\prime}(x, \lambda)}{\tilde{\psi}(x, \lambda)}$. Hence, from equation (1) and transmission conditions (4)-(7), $q(x)=\tilde{q}(x)$, a.e., $\theta_{i}=\tilde{\theta}_{i}, \gamma_{i}=\tilde{\gamma}_{i}, i=3-6$, and from (9) and (10), $\theta_{i}=\tilde{\theta}_{i}$, $\theta_{j}^{\prime}=\tilde{\theta}_{j}^{\prime}, \gamma_{j}^{\prime}=\tilde{\gamma}_{j}^{\prime}, i, j=1,2$.

Lemma 6 The following representation holds

$$
M(\lambda)=\sum_{n=0}^{\infty} \frac{\alpha_{1} \alpha_{2}}{\mu_{n}\left(\lambda_{n}-\lambda\right)}
$$

Proof Weyl function $M(\lambda)$ is a meromorphic function with respect to $\lambda$, which has simple poles at $\lambda_{n}$. Therefore, we calculate

$$
\operatorname{Re}_{\lambda=\lambda_{n}} s(\lambda)=\frac{\theta_{2}^{\prime} \psi^{\prime}\left(a, \lambda_{n}\right)-\theta_{1}^{\prime} \psi\left(a, \lambda_{n}\right)}{\beta_{1} \dot{\Delta}\left(\lambda_{n}\right)} .
$$

Since $\kappa_{n}=\frac{\theta_{2}^{\prime} \psi^{\prime}\left(a, \lambda_{n}\right)-\theta_{1}^{\prime} \psi\left(a, \lambda_{n}\right)}{\beta_{1}}$ and $\dot{\Delta}\left(\lambda_{n}\right)=\frac{\kappa_{n} \mu_{n}}{\alpha_{1} \alpha_{2}}$,

$$
\operatorname{Re}_{\lambda=\lambda_{n}} M(\lambda)=\frac{\alpha_{1} \alpha_{2}}{\mu_{n}} .
$$

Let $\Gamma_{N}=\left\{\lambda: \lambda=k^{2},|k|=\sqrt{\lambda_{N}}+\varepsilon\right\}$, where $\varepsilon$ is a sufficiently small number. Consider the contour integral $I_{N}(\lambda)=\frac{1}{2 \pi i} \int_{\Gamma_{n}} \frac{M(\mu)}{\mu-\lambda} d \mu, \lambda \in \operatorname{int} \Gamma_{N}$. For $\lambda \in G_{w}$,

$$
\Delta(\lambda) \geq|\lambda|^{7 / 2} C_{w} e^{|\operatorname{Im} k|\left(\left(\delta_{1}-a\right) \rho_{1}+\left(\delta_{2}-\delta_{1}\right) \rho_{2}+\left(b-\delta_{2}\right) \rho_{3}\right)}
$$

satisfies. Using this equality and (16), we get

$$
|M(\lambda)| \leq \frac{C_{w}}{|\lambda|} \quad \text { for } \lambda \in G_{w}
$$


Thus, $\lim _{N \rightarrow \infty} I_{N}(\lambda)=0$. As a result, the residue theorem and (23) yield

$$
M(\lambda)=\sum_{n=0}^{\infty} \frac{\alpha_{1} \alpha_{2}}{\mu_{n}\left(\lambda_{n}-\lambda\right)} .
$$

Theorem 4 If $\lambda_{n}=\tilde{\lambda}_{n}$ and $\mu_{n}=\tilde{\mu}_{n}$ for all $n$, then $L \equiv \tilde{L}$, i.e., $q(x)=\tilde{q}(x)$, a.e., $\theta_{i}=\tilde{\theta}_{i}, \gamma_{i}=\tilde{\gamma}_{i}$, $i=1-6, \delta_{j}=\tilde{\delta}_{j}, \theta_{j}^{\prime}=\tilde{\theta}_{j}^{\prime}, \gamma_{j}^{\prime}=\tilde{\gamma}_{j}^{\prime}, j=1,2$. Hence, problem (1)-(7) is uniquely determined by spectral data $\left\{\lambda_{n}, \mu_{n}\right\}$.

Proof If $\lambda_{n}=\tilde{\lambda}_{n}$ and $\mu_{n}=\tilde{\mu}_{n}$ for all $n$, then $M(\lambda)=\tilde{M}(\lambda)$ by Lemma 6 . Therefore, we get $L=\tilde{L}$ by Theorem 3 .

Let us consider the boundary value problem $L_{1}$ that we get the condition $\theta_{2}^{\prime} y^{\prime}(a, \lambda)-$ $\theta_{1}^{\prime} y(a, \lambda)=0$ instead of condition (2) in $L$. Let $\left\{\tau_{n}\right\}_{n \geq 0}$ be the eigenvalues of the problem $L_{1}$. It is clear that $\tau_{n}$ are zeros of

$$
\Delta_{1}(\tau):=\theta_{2}^{\prime} \psi^{\prime}(a, \tau)-\theta_{1}^{\prime} \psi(a, \tau)
$$

Theorem 5 If $\lambda_{n}=\tilde{\lambda}_{n}$ and $\tau_{n}=\tilde{\tau}_{n}$ for all n, then $L\left(q, \delta_{j}, \theta_{k}, \gamma_{i}, \gamma_{j}^{\prime}\right)=L\left(\tilde{q}, \tilde{\delta}_{j}, \tilde{\theta}_{k}, \tilde{\gamma}_{i}, \tilde{\gamma}_{j}^{\prime}\right), i=1-6$, $k=3-6, j=1,2$.

Hence, the problem $L$ is uniquely determined by the sequences $\left\{\lambda_{n}\right\}$ and $\left\{\tau_{n}\right\}$, except coefficients $\theta_{j}$ and $\theta_{j}^{\prime}$.

Proof Since the characteristic functions $\Delta(\lambda)$ and $\Delta_{1}(\tau)$ are entire of order $\frac{1}{2}$, functions $\Delta(\lambda)$ and $\Delta_{1}(\tau)$ are uniquely determined up to multiplicative constant with their zeros by Hadamard's factorization theorem [37]

$$
\begin{aligned}
& \Delta(\lambda)=C \prod_{n=0}^{\infty}\left(1-\frac{\lambda}{\lambda_{n}}\right), \\
& \Delta_{1}(\tau)=C_{1} \prod_{n=0}^{\infty}\left(1-\frac{\tau}{\tau_{n}}\right),
\end{aligned}
$$

where $C$ and $C_{1}$ are constants dependent on $\left\{\lambda_{n}\right\}$ and $\left\{\tau_{n}\right\}$, respectively. Therefore, when $\lambda_{n}=\tilde{\lambda}_{n}$ and $\tau_{n}=\tilde{\tau}_{n}$ for all $n, \Delta(\lambda) \equiv \tilde{\Delta}(\lambda)$ and $\Delta_{1}(\tau) \equiv \tilde{\Delta}_{1}(\tau)$. Hence, $\theta_{2}^{\prime} \psi^{\prime}(a, \tau)-$ $\theta_{1}^{\prime} \psi(a, \tau)=\tilde{\theta}_{2}^{\prime} \tilde{\psi}^{\prime}(a, \tau)-\tilde{\theta}_{1}^{\prime} \tilde{\psi}(a, \tau)$. As a result, we get $M(\lambda)=\tilde{M}(\lambda)$ by (16). So, the proof is completed by Theorem 3 .

\section{Competing interests \\ The author declares that he has no competing interests.}

Received: 18 June 2013 Accepted: 26 August 2013 Published: 11 September 2013

\section{References}

1. Fulton, CT: Two-point boundary value problems with eigenvalue parameter contained in the boundary conditions. Proc. R. Soc. Edinb. A 77, 293-308 (1977)

2. Kobayashi, M: Eigenvalues of discontinuous Sturm-Liouville problems with symmetric potentials. Comput. Math. Appl. 18(4), 355-364 (1989)

3. Likov, AV, Mikhailov, YA: The Theory of Heat and Mass Transfer. Qosenergaizdat (1963)

4. Shkalikov, AA: Boundary value problems for ordinary differential equations with a parameter in boundary conditions. Tr. Semin. Im. I.G. Petrovskogo 9, 190-229 (1983)

5. Binding, PA, Browne, PJ, Seddighi, K: Sturm-Liouville problems with eigenparameter dependent boundary conditions. Proc. Edinb. Math. Soc. 37, 57-72 (1993) 
6. Binding, PA, Browne, PJ, Watson, BA: Inverse spectral problems for Sturm-Liouville equations with eigenparameter dependent boundary conditions. J. Lond. Math. Soc. 62, 161-182 (2000)

7. Binding, PA, Browne, PJ, Watson, BA: Equivalence of inverse Sturm-Liouville problems with boundary conditions rationally dependent on the eigenparameter. J. Math. Anal. Appl. 291, 246-261 (2004)

8. Fulton, CT: Singular eigenvalue problems with eigenvalue parameter contained in the boundary conditions. Proc. R. Soc. Edinb. A 87, 1-34 (1980)

9. Mennicken, R, Schmid, H, Shkalikov, AA: On the eigenvalue accumulation of Sturm-Liouville problems depending nonlinearly on the spectral parameter. Math. Nachr. 189, 157-170 (1998)

10. Russakovskii, EM: Operator treatment of boundary problems with spectral parameters entering via polynomials in the boundary conditions. Funct. Anal. Appl. 9, 358-359 (1975)

11. Schmid, H, Tretter, C: Singular Dirac systems and Sturm-Liouville problems nonlinear in the spectral parameter. J. Differ. Equ. 181(2), 511-542 (2002)

12. Ozkan, AS, Keskin, B: Spectral problems for Sturm-Liouville operator with boundary and jump conditions linearly dependent on the eigenparameter. Inverse Probl. Sci. Eng. 20, 799-808 (2012)

13. Akdoğan, Z, Demirci, M, Mukhtarov, OS: Sturm-Liouville problems with eigendependent boundary and transmissions conditions. Acta Math. Sci. 25(4), 731-740 (2005)

14. Akdoğan, Z, Demirci, M, Mukhtarov, OS: Discontinuous Sturm-Liouville problem with eigenparameter-dependent boundary and transmission conditions. Acta Appl. Math. 86, 329-334 (2005)

15. Binding, PA, Browne, PJ, Watson, BA: Sturm-Liouville problems with boundary conditions rationally dependent on the eigenparameter II. J. Comput. Appl. Math. 148, 147-169 (2002)

16. Kerimov, NB, Memedov, KK: On a boundary value problem with a spectral parameter in the boundary conditions. Sib. Mat. Zh. 40(2), 325-335 (1999). (English translation: Sib. Math. J. 40(2), 281-290 (1999))

17. Mukhtarov, OS, Kadakal, M, Muhtarov, FS: On discontinuous Sturm-Liouville problem with transmission conditions. J. Math. Kyoto Univ. 444, 779-798 (2004)

18. Tunç, E, Muhtarov, OS: Fundamental solution and eigenvalues of one boundary value problem with transmission conditions. Appl. Math. Comput. 157, 347-355 (2004)

19. Yakubov, S: Completeness of Root Functions of Regular Differential Operators. Longman, New York (1994)

20. Meschanov, VP, Feldstein, AL: Automatic Design of Directional Couplers. Sviaz, Moscow (1980)

21. Tikhonov, AN, Samarskii, AA: Equations of Mathematical Physics. Pergamon, Oxford (1990)

22. Voitovich, NN, Katsenelbaum, BZ, Sivov, AN: Generalized Method of Eigen-Vibration in the Theory of Diffraction. Nauka, Moscow (1997)

23. McNabb, A, Anderssen, R, Lapwood, E: Asymptotic behavior of the eigenvalues of a Sturm-Liouville system with discontinuous coefficients. J. Math. Anal. Appl. 54, 741-751 (1976)

24. Shieh, CT, Yurko, VA: Inverse nodal and inverse spectral problems for discontinuous boundary value problems. J. Math. Anal. Appl. 347, 266-272 (2008)

25. Willis, C: Inverse Sturm-Liouville problems with two discontinuities. Inverse Probl. 1, $263-289$ (1985)

26. Yang, CF: An interior inverse problem for discontinuous boundary-value problems. Integral Equ. Oper. Theory 65 , 593-604 (2009)

27. Yang, CF, Yang, XP: An interior inverse problem for the Sturm-Liouville operator with discontinuous conditions. Appl. Math. Lett. 22, 1315-1319 (2009)

28. Yang, Q, Wang, W: Asymptotic behavior of a differential operator with discontinuities at two points. Math. Methods Appl. Sci. 34, 373-383 (2011)

29. Yurko, VA: Integral transforms connected with discontinuous boundary value problems. Integral Transforms Spec. Funct. 10, 141-164 (2000)

30. Litvinenko, ON, Soshnikov, Vl: The Theory of Heterogenious Lines and Their Applications in Radio Engineering. Moscow (1964)

31. Shepelsky, DG: The inverse problem of reconstruction of the medium's conductivity in a class of discontinuous and increasing functions. In: Spectral Operator Theory and Related Topics. Adv. Soviet Math., vol. 19, pp. 209-232. Am. Math. Soc., Providence (1994)

32. Anderssen, RS: The effect of discontinuities in density and shear velocity on the asymptotic overtone structure of torsional eigenfrequencies of the earth. Geophys. J. R. Astron. Soc. 50, 303-309 (1997)

33. Lapwood, FR, Usami, T: Free Oscillations of the Earth. Cambridge University Press, Cambridge (1981)

34. Ambarsumian, VA: Über eine frage der eigenwerttheorie. Z. Phys. 53, 690-695 (1929)

35. Borg, G: Eine umkehrung der Sturm-Liouvilleschen eigenwertaufgabe. Bestimmung der differentialgleichung durch die eigenwerte. Acta Math. 78, 1-96 (1946)

36. Zhdanovich, VF: Formulae for the zeros of Dirichlet polynomials and quasi-polynomials. Dokl. Akad. Nauk SSSR 135(8), 1046-1049 (1960)

37. Titchmarsh, EC: The Theory of Functions. Oxford University Press, London (1939)

doi:10.1186/1687-2770-2013-209

Cite this article as: Güldü: Inverse eigenvalue problems for a discontinuous Sturm-Liouville operator with two

discontinuities. Boundary Value Problems 2013 2013:209. 